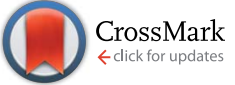

Cite this: Chem. Sci., 2016, 7, 2342

\title{
Simple and ultrastable all-inclusive pullulan tablets for challenging bioassays $\uparrow$
}

\author{
Sana Jahanshahi-Anbuhi,,$^{\text {ab }}$ Balamurali Kannan, $\star^{a}$ Vincent Leung, ${ }^{b}$ Kevin Pennings, ${ }^{b}$ \\ Meng Liu, ${ }^{a c}$ Carmen Carrasquilla, ${ }^{a}$ Dawn White, ${ }^{a}$ Yingfu Li, ${ }^{\text {ac }}$ Robert $\mathrm{H}$. Pelton, ${ }^{\text {ab }}$ \\ John D. Brennan ${ }^{\star a}$ and Carlos D. M. Filipe ${ }^{\star a b}$
}

\begin{abstract}
Many biodetection systems employ labile enzymes and substrates that need special care, making it hard to routinely use them for point-of-care or field applications. In this work we provide a simple solution to this challenging problem through the creation of all-inclusive pullulan assay tablets. The proposed tablet system not only enhances the long-term stability of both enzymes and organic substrates, but also simplifies the assay procedure. The enhanced stability is attributed to two factors: the restriction of the molecular motion of proteins and impermeability to molecular oxygen afforded by the tables. These tablets dissolve rapidly upon addition to testing samples, making the test very easy to perform. Using the ATPdetecting luciferase-luciferin system as an example, we show that the tablet-based assay can achieve highly sensitive detection of ATP in biological samples and that the activity of the assay tablets remains unchanged for over a month at room temperature.
\end{abstract}

Received 3rd November 2015 Accepted 1st January 2016

DOI: $10.1039 / \mathrm{c} 5 \mathrm{sc} 04184 \mathrm{~h}$

www.rsc.org/chemicalscience stabilize this enzyme have included the use of osmolytes/ stabilizers, ${ }^{23}$ immobilization of luciferase on solid supports such as pre-coated polymer films, ${ }^{24}$ dendrimers ${ }^{25}$ and glass rods, ${ }^{26}$ and entrapment into sol-gel derived silica. ${ }^{27-30}$ Although these methods are useful in slowing the loss of luciferase activity, they are not effective in maintaining the long-term storage stability of luciferase. In addition, most of these methods involve very complex procedures, making them of limited practical use. Furthermore, none of these methods address the instability issue of luciferin, which is prone to degradation as a result of oxidation. ${ }^{31}$

Recently, our groups reported on a new method for stabilizing labile enzymes and substrates by entrapment into pullulan, a polysaccharide produced by the fungus Aureobasidium pullulans. ${ }^{32,33}$ Herein, we extend the study of pullulan-entrapped reagents to the very labile luciferase/luciferin assay system, and investigate whether an "all-inclusive" tablet containing all required reagents and co-factors (luciferase, luciferin, $\mathrm{MgSO}_{4}$, $\mathrm{MgCO}_{3}$, dithiothreitol (DTT), ethylenediaminetetraaceticacid (EDTA), co-enzyme A (CoA) and tricine) could be used for facile detection of ATP using a mix-and-read assay. Pullulan tablets were prepared by casting and drying a 10\% (final concentration) pullulan solution containing all seven components at defined concentrations. Optimal intensity and stability of luminescence were achieved by using a $\mathrm{pH}$ of 7.8 , as reported previously in the literature. ${ }^{34,35}$ In addition, the buffer was added directly to the tablet formulation to ensure that the $\mathrm{pH}$ was maintained at a constant value during drying and vitrification. These formed optically clear, glass-like materials that could be easily handled (Fig. 1). 


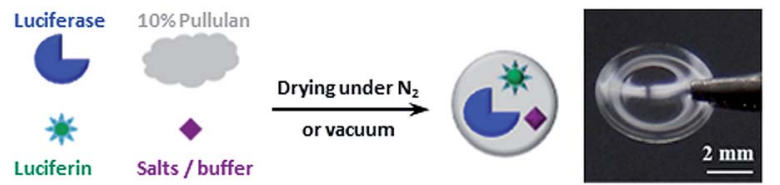

Fig. 1 Method to produce all-inclusive pullulan assay tablets for ATP detection. A photograph of an all-inclusive pullulan tablet is shown on the right, where the tablet is a disc-shaped film with a diameter of 6 $\mathrm{mm}$.

\section{Results and discussion}

Initial studies focused on the thermal stability of the luciferase within the pullulan tablets relative to solution (Fig. 2A). It was found that the entrapped enzyme maintained similar activity in the tested temperature range $\left(22-65{ }^{\circ} \mathrm{C}\right)$, suggesting that the pullulan entrapment method is useful for conducting assays for field applications that often have varying ambient temperatures. In contrast, the solution-based assay mixture began to lose activity even at $30{ }^{\circ} \mathrm{C}$ and became completely inactive at $50{ }^{\circ} \mathrm{C}$.

To probe into the mechanisms of stabilization, we examined the steady-state emission spectra of luciferase and the model protein human serum albumin (HSA). Also, the steady-state fluorescence anisotropy of HSA was measured to probe protein conformation and dynamics. HSA was chosen as a surrogate for luciferase for dynamics studies because it contains a single tryptophan, which is required for analysis of anisotropy data, and because the relationship between HSA dynamics and conformation is well understood. ${ }^{36}$ Steady-state emission spectra for luciferase and HSA are shown in Fig. S1. $\dagger$ Both proteins show identical spectra in aqueous solution and in
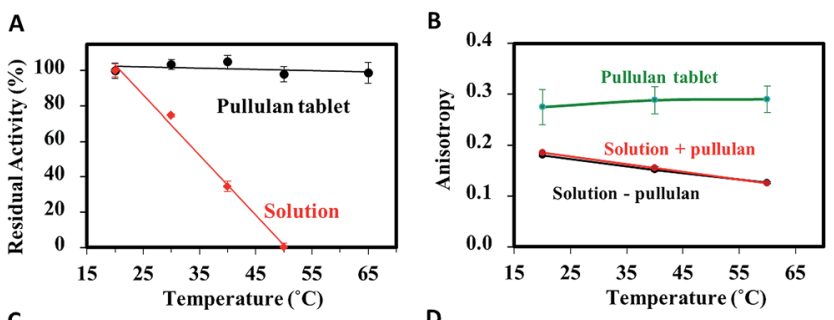

C

D
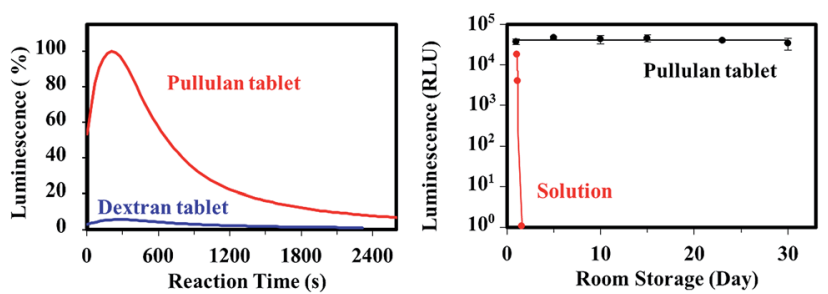

Fig. 2 (A) Thermal stability of all-inclusive tablet vs. solution. (B) Steady-state anisotropy of Trp within HSA in pullulan tablet, pullulan solution and buffer solution. (C) Comparison of luminescence of allinclusive pullulan vs. dextran tablets cast under ambient conditions (refer to Fig. S5† for the data obtained when luciferase and luciferin were included in separate pullulan tablets). (D) Long-term stability of pullulan tablet produced under nitrogen vs. solution. solutions with $10 \%(\mathrm{w} / \mathrm{v})$ pullulan, showing that pullulan has no effect on protein conformation. In the pullulan tablet, both proteins show small blue-shifts in the emission maximum, but retain the same overall spectral contour. These data show that the entrapped proteins do not aggregate, and that the Trp residue is in an environment where molecular motion is restricted, and thus dipolar solvent relaxation cannot occur. ${ }^{37}$ As shown in Fig. 2B, the steady-state anisotropy value of pullulanentrapped HSA $(\sim 0.29)$ remains close to the limiting anisotropy value $(0.31)$ even up to $60^{\circ} \mathrm{C}$, whereas the anisotropy of HSA in solution starts at a lower value (0.18) and drops by a factor of $\sim 2$ at $60{ }^{\circ} \mathrm{C}$. This further demonstrates that the rotational motion of the entrapped protein and the surrounding pullulan molecules is highly restricted, consistent with the inability of protein to unfold within pullulan sugar-glass. ${ }^{38}$ The restricted molecular motion is thus likely to be a major contributor to the enhanced stability of luciferase in pullulan tablets. In addition, the high glass transition temperature of pullulan glass $\left(T_{\mathrm{g}}>100{ }^{\circ} \mathrm{C}\right)$ is likely to be a contributing factor as well, as it aids in maintaining the restricted mobility of the entrapped enzyme even at high temperatures. ${ }^{39}$

Further analysis of the effect of pullulan on other assay components (Fig. S2 $\dagger$ ) demonstrates that the presence of the sugar does not affect the signal generated by the assay, demonstrating that pullulan is an inert polymer that does not interact with any of the components used in the assay. An unexpected feature of pullulan tablet is the ability to better protect labile reagents compared with other sugar-glasses, such as those formed from dextran, a structurally similar polysaccharide. Production of pullulan and dextran tablets was done in ambient air and at room temperature (simplest possible method for casting) and these were dried for $24 \mathrm{~h}$. As shown in Fig. 2C, the pullulan tablets both preserved the activity of luciferase and prevented the oxidation of luciferin, as evidenced by the increase in light emission associated with the dissolution of the tablets in the presence of ATP. In contrast, the dextran tablets produced $<10 \%$ of the emission intensity, indicative of a much lower activity of luciferase and/or significant oxidation of luciferin. Direct inhibition of luciferase by dextran was ruled out as the luminescent assay showed full activity in fresh dextran solutions (Fig. S3†). These results indicate that pullulan has a highly unique ability to stabilize luciferase and protect luciferin during the tablet forming (i.e. drying) process. We also created polyethylene glycol (PEG, MW 6000) tablets (see ESI $\dagger$ ) containing luciferase and luciferin, but no signal was obtained when performing the assay with these films, further indicating that pullulan has a unique ability to stabilized (bio)molecules.

Further evaluation of assay performance over time revealed that the production of pullulan tablets under ambient conditions resulted in poor long-term stability, likely owing to oxidation of luciferin by oxygen that was entrapped in the tablets. This hypothesis was verified through mass spectrometry, which showed that dehydroluciferin was indeed formed in such tablets (data not shown). To avoid this problem, assay tablets were produced under nitrogen and dried under vacuum. As shown in Fig. 2D, these pullulan tablets retained high activity $(>97 \%)$ when stored at room temperature for up to 30 days, 
whereas the assay components in solution lost all activity within a few hours. These results further confirm the observation that pullulan films are oxygen-impermeable. ${ }^{32}$ Interestingly, entrapment of luciferase alone (Fig. S4 $\dagger$ ) resulted in a loss of $20 \%$ activity in 30 days, demonstrating that the presence of the additional assay components helped to stabilize the entrapped enzyme (Fig. 2D).

Important issues when using reagent tablets are the degree to which the formulation can be manipulated to optimize the response of the assay, and how the rate of dissolution might affect the overall output from the assay. To address the first issue, we examined tablets prepared with varying levels of $\mathrm{MgSO}_{4}, \mathrm{MgCO}_{3}$ and CoA. Variations in the levels of the salts had a minor effect on the assay performance (data not shown), while alterations in CoA concentration had a significant effect. The role of CoA in the luciferase-luciferin assay is to react with dehydroluciferyladenylate (L-AMP), a byproduct formed in the presence of oxygen and a potent inhibitor of luciferase, ${ }^{40}$ generating luciferyl-coenzyme A (L-CoA) through thiolysis with CoA. L-CoA is a much weaker inhibitor of luciferase than LAMP, hence adding CoA to the assay substantially increases signal generation. As shown in Fig. 3A, increasing CoA concentration significantly improved assay sensitivity, in agreement with results obtained in solution-based assays. ${ }^{34}$ As shown in Fig. 3B, when using optimal amounts of CoA, the tablet based assay has sensitivity that is similar to solution, though the maximum intensity decreases somewhat (ca. 22\%). On the other hand, the maximum intensity occurs later owing to the dissolution time of the tablet, making it easier to detect the maximum signal reproducibly. It is worth noting that following the attainment of the maximum signal; the rate at which the signal decreases is virtually identical to that of solution-based assay. In addition, the integrated intensity of the tablet-based assay is within $10 \%$ of the solution assay.

\section{A}

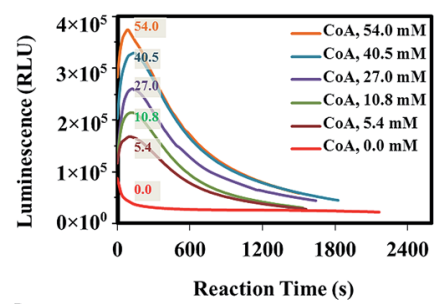

B

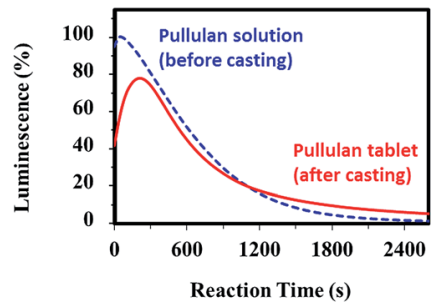

Fig. 3 (A) Effect of CoA concentration on assay performance (values are final concentrations after tablet dissolution). $54 \mathrm{mM}$ represents the optimal concentration of CoA in our all-inclusive assay system. (B) Comparison of signal evolution from a tablet-based assay and a solution assay.
The ability of luminescent tablets to detect free and cellbased ATP is demonstrated in Fig. 4. Luminescence (RLU) was measured as a function of ATP concentration in tricine buffer. The limit of detection was calculated to be $5.6 \mathrm{pM}$ at $3 \sigma$ above the background, which corresponds to an ATP concentration of 5 attomoles per $\mu \mathrm{L}$. This value is comparable to the ultrasensitive ATP detection limit reported using sol-gel entrapped luciferase and $\sim 500$ times better than a recently reported voltammetric ATP detection method. ${ }^{41}$

The ability of the all-inclusive pullulan tablets to detect the ATP from lysed $E$. coli cells was also tested (Fig. 4B). The detection limit was found to be $3 \times 10^{3}$ cells per $\mathrm{mL}$ for the lysed cells, which was close to the LOD for a highly sensitive commercially available assay kit $\left(2 \times 10^{3}\right.$ cells per $\left.\mathrm{mL}\right) .^{\mathbf{4 2}}$ However, only the all-inclusive tablet method provided a simple, one-step assay format that was both reproducible and user-friendly. By contrast, the Promega BacTiter-Glo ${ }^{\mathrm{TM}}$ technology involved preparing a substrate solution from a freeze-dried substrate and then mixing with a buffer solution where both the buffer and substrate required storage at $-20{ }^{\circ} \mathrm{C}$.

In addition to the ATP assay using the luciferase/luciferin system, we expect that pullulan tablets should also be useful for the fabrication of highly stable bioassay kits containing other fragile biomolecules and enzymes. This has already been demonstrated for pesticide analysis using acetylcholinesterase and the indoxyl acetate substrate, ${ }^{32}$ but should be capable of providing a generic platform for point-of-care (POC) diagnostics. Such tablet-based assays could significantly improve health-care support in resource-limited settings, and allow multiple applications across various fields such as environmental monitoring, food safety, and clinical diagnostics that require the use of labile (bio)reagents. This strategy should also decrease the number of steps needed to complete an analysis and the degree of user intervention in current multistep assays.

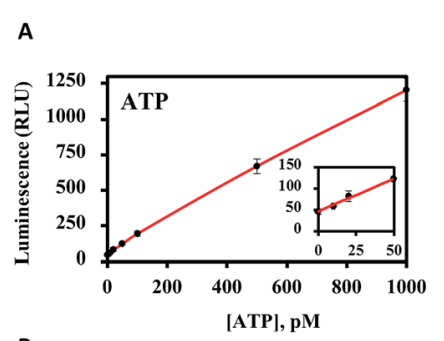

B

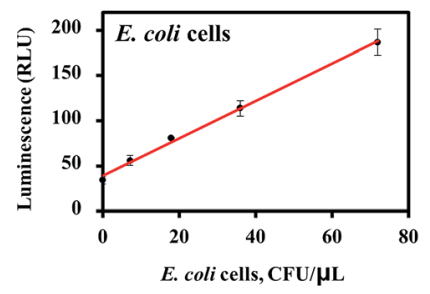

Fig. 4 (A) ATP detection using all-inclusive pullulan tablets. (B) Detection of lysed $E$. coli cells using the all-inclusive luminescent tablets. 


\section{Conclusions}

In conclusion, we have succeeded in entrapping the highly unstable luciferase-luciferin system into pullulan tablets to produce a highly stable, all-inclusive ATP assay system with excellent reproducibility and user-friendliness. The basis of the enhanced stability is shown to be a combination of restricted molecular motion of the entrapped protein, and oxygen impermeability, which maintains the molecular structure of oxygen-sensitive reagents. The dissolution rate of pullulan delays the onset of maximum signal evolution, which makes ATP detection more reproducible. It is noteworthy that the method for producing these tablets is particularly cost-effective ( $\sim 1$ USD per 100 tablets). Given the importance of ATP detection, it is conceivable that the all-inclusive tablets can be adopted for a broad range of applications ranging from inexpensive on-site ATP detection to high-throughput kinase activity analysis where simplicity, sensitivity and stable light emission are desired.

\section{Acknowledgements}

The authors thank the Natural Sciences and Engineering Research Council of Canada for funding through the SENTINEL Bioactive Paper Network. We also thank the Canadian Foundation for Innovation and the Ontario Ministry of Research and Innovation for Infrastructure funding to the Biointerfaces Institute. JDB holds the Canada Research Chair in Bioanalytical Chemistry and Biointerfaces. RP holds the Canada Research Chair in Interfacial Technologies. Y. L. holds the Canada Research Chair in Chemical Biology of Nucleic Acids.

\section{Notes and references}

1 O. Kepp, L. Galluzzi, M. Lipinski, J. Yuan and G. Kroemer, Nat. Rev. Drug Discovery, 2011, 10, 221-237.

2 V. Gubala, L. F. Harris, A. J. Ricco, M. X. Tan and D. E. Williams, Anal. Chem., 2012, 84, 487-515.

3 M. S. Luchansky and R. C. Bailey, Anal. Chem., 2012, 84, 793821.

4 S. Jahanshahi-Anbuhi, K. Pennings, V. Leung, B. Kannan, J. D. Brennan, C. D. M. Filipe and R. Pelton, ACS Appl. Mater. Interfaces, 2015, 7, 25434-25440.

5 M. Liu, C. Hui, Q. Zhang, J. Gu, B. Kannan, S. JahanshahiAnbuhi, C. D. M. Filipe and J. D. Brennan, Angew. Chem., Int. Ed., 2015, 1-5.

6 S. Jahanshahi-Anbuhi, A. Henry, V. Leung, C. Sicard, K. Pennings, R. Pelton, J. D. Brennan and C. D. M. Filipe, Lab Chip, 2014, 14, 229-236.

7 I. D. Odell and D. Cook, J. Invest. Dermatol., 2013, 133, 4.

8 D. Zhanga, Y. Lua, J. Jiangb, Q. Zhanga, Y. Yaoa, P. Wanga, B. Chenc, Q. Chengc, G. L. Liub and Q. Liu, Biosens. Bioelectron., 2015, 67, 237-242.

9 Y. Z. Lin, T. L. Chang and C. C. Chang, Sens. Actuators, B, 2014, 190, 486-493.

10 N. N. Ugarova, Nat. Photonics, 2008, 2, 8-9.
11 E. H. White, F. McCapra, G. F. Field and W. D. McElroy, J. Am. Chem. Soc., 1961, 83, 2402-2403.

12 T. Goto and Y. Kishi, Angew. Chem., Int. Ed. Engl., 1968, 7, 407-414.

13 E. Amodio and C. Dino, J. Infect. Public Health, 2014, 7, 9298.

14 Y. Wang, L. Tang, Z. Li, Y. Lin and J. Li, Nat. Protoc., 2014, 9, 1944-1955.

15 N. Omidbakhsh, F. Ahmadpour and N. Kenny, PLoS One, 2014, 9, 1-8.

16 S. Falzoni, G. Donvito and F. Di Virgilio, Interface Focus, 2013, 3, 1-8.

17 X. He, Z. Li, X. Jia, K. Wang and J. Yin, Talanta, 2013, 111, 105-110.

18 S. B. Kim, M. Torimura and H. Tao, Bioconjugate Chem., 2013, 24, 2067-2075.

19 J. A. Cruz-Aguado, Y. Chen, Z. Zhang, N. H. Elowe, M. A. Brook and J. D. Brennan, J. Am. Chem. Soc., 2004, 126, 6878-6879.

20 M. R. Ganjalikhany, B. Ranjbar, S. Hosseinkhani, K. Khalifeh and L. Hassani, J. Mol. Catal. B: Enzym., 2010, 62, 127-132.

21 M. Baker, Nat. Methods, 2012, 9, 225.

22 R. Herbst, U. Schafer and R. Seckler, J. Biol. Chem., 1997, 272, 7099-7105.

23 J. K. Kaushik and R. Bhat, J. Biol. Chem., 2003, 278, 2645826465.

24 N. Y. Filippova, A. F. Dukhovich and N. N. Ugarova, J. Biolumin. Chemilumin., 1989, 4, 419-422.

25 S. A. Miller, E. D. Hong and D. Wright, Macromol. Biosci., 2006, 6, 839-845.

26 Y. Lee, I. Jablonski and M. Deluca, Anal. Biochem., 1977, 80, 496-501.

27 T. R. Besanger and J. D. Brennan, J. Sol-Gel Sci. Technol., 2006, 40, 209-225.

28 Y. Li and W. T. Yip, J. Am. Chem. Soc., 2005, 127, 1275612757.

29 T. Y. Lin, C. H. Wu and J. D. Brennan, Biosens. Bioelectron., 2007, 22, 1861-1867.

30 X. H. Sui, J. A. Cruz-Aguado, Y. Chen, Z. Zhang, M. A. Brook and J. D. Brennan, Chem. Mater., 2005, 17, 1174-1182.

31 S. Inouye, Methods Enzymol., 2000, 326, 165-174.

32 S. Jahanshahi-Anbuhi, K. Pennings, V. Leung, M. Liu, C. Carrasquilla, B. Kannan, Y. Li, R. Pelton, J. D. Brennan and C. D. M. Filipe, Angew. Chem., Int. Ed., 2014, 53, 61556158.

33 B. Kannan, S. Jahanshahi-Anbuhi, R. Pelton, Y. Li, C. D. M. Filipe and J. D. Brennan, Anal. Chem., 2015, 87, 9288-9293.

34 E. S.-v. Olst, C. Vermeulen, R. X. d. Menezes, M. Howell, E. F. Smit and V. W. v. Beusechem, J. Biomol. Screening, 2012, 1, 1-9.

35 Sigma-Aldrich, Luciferase from Photinus pyralis (firefly), Catalog ID \# L9506.

36 K. Flora, J. D. Brennan, G. A. Baker, M. A. Doody and F. V. Bright, Biophys. J., 1998, 75, 1084-1096.

37 J. R. Lakowicz, Principles of Fluorescence Spectroscopy, Kluwer Academic/Plenum, New York, 2nd edn, 1999. 
38 M. T. Cicerone and C. L. Soles, Biophys. J., 2004, 86, 38363845.

39 B. S. Chang, R. M. Beauvais, A. Dong and J. F. Carpenter, Arch. Biochem. Biophys., 1996, 331, 249-258.
40 L. P. d. Silvaa and J. C. G. E. d. Silva, Photochem. Photobiol. Sci., 2011, 10, 1039-1045.

41 Y. Guo, X. Sun, G. Yang and J. Liu, Chem. Commun., 2014, 50, 7659-7662.

42 Promega: TB337 2012, 1. 